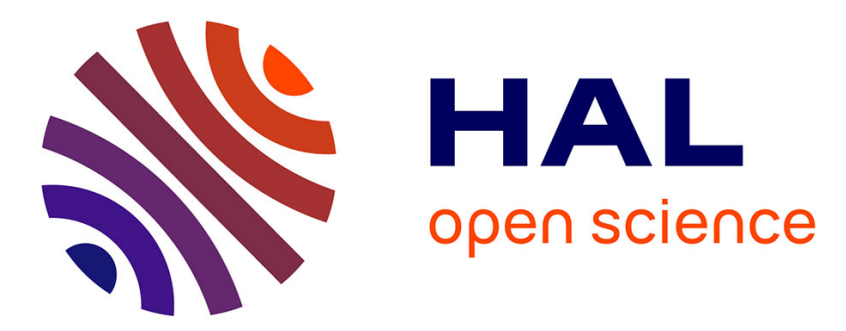

\title{
Prospects for strangeness and charm measurements with the CBM experiment
}

\author{
Volker Friese
}

\section{To cite this version:}

Volker Friese. Prospects for strangeness and charm measurements with the CBM experiment. Journal of Physics G: Nuclear and Particle Physics, 2010, 37 (9), pp.94025. 10.1088/0954-3899/37/9/094025 . hal-00600844

\section{HAL Id: hal-00600844 https://hal.science/hal-00600844}

Submitted on 16 Jun 2011

HAL is a multi-disciplinary open access archive for the deposit and dissemination of scientific research documents, whether they are published or not. The documents may come from teaching and research institutions in France or abroad, or from public or private research centers.
L'archive ouverte pluridisciplinaire HAL, est destinée au dépôt et à la diffusion de documents scientifiques de niveau recherche, publiés ou non, émanant des établissements d'enseignement et de recherche français ou étrangers, des laboratoires publics ou privés. 


\title{
Prospects for strangeness and charm measurements with the CBM experiment
}

\author{
Volker Friese \\ GSI Helmholtzzentrum für Schwerionenforschung, Darmstadt, Germany \\ E-mail: v.friese@gsi.de
}

\begin{abstract}
.
The CBM experiment will investigate strongly interacting matter at extreme densities at the future accelerator facility FAIR in Darmstadt, in search for the first-order phase transition from confined to deconfined matter. Key observables of CBM are strange and charmed hadrons. We discuss the performance of the planned experimental setup with respect to the measurement of strange and multi-strange hadrons, open charm and charmonium, based on detailed detector simulations which include a realistic detector response.
\end{abstract}




\section{The CBM experiment}

The interest in the high-density part of the QCD phase diagram and its features, namely the possible first-order phase transition from confined to deconfined matter, the critical endpoint separating the first-order transition region from that of a cross-over at lower densities, and the properties of hadrons in a dense environment, reflect in several experimental activities in accelerator laboratories around the world: the RHIC energy scan programme, the NA61 activities at CERN-SPS, the NICA project at JINR Dubna, and the CBM project at the future facility FAIR in Darmstadt [1]. Amongst them, the fixed-target experiment CBM provides the unique opportunity to study extremely rare probes like charmed hadrons, which are close to their production thresholds at FAIR beam energies $(10-45 \mathrm{~A} \mathrm{GeV})$. This opportunity is due to the high beam intensities to be delivered by the SIS-300 synchrotron of FAIR (up to $10^{9}$ ions/s), giving rise to interaction rates of up to $10 \mathrm{MHz}$ with a typical $1 \%$ interaction target.

$\mathrm{CBM}$ is being designed to measure hadronic, leptonic and photonic observables in a large acceptance, covering the full $p_{t}$ range and rapidities from centre-of-mass rapidity close to beam rapidity. The planned experimental setup is shown in the left panel of Fig. 1. The core tracker (STS), consisting of eight stations of Silicon microstrip detectors, is located between the yokes of a super-conducting dipole magnet. Close to the target, the Micro-Vertex Detector (MVD) provides excellent resolution of displaced vertices from open charm decays. Downstream of the STS, a RICH and several layers of Transition Radiation Detectors (TRD) provide electron identification in a large momentum range. The TRD also serves for global tracking, connecting the tracks reconstructed in the STS with the time-of-flight wall located approximately $10 \mathrm{~m}$ downstream of the target. An electromagentic calorimeter after the TOF detector enables the measurement of neutral particles. The setup is completed with a downstream calorimeter (PSD) for event characterisation in terms of centrality and reaction plane angle.

For muon measurements, the RICH will be replaced by an absorber system interlayed with several tracking detector planes, allowing to follow the tracks reconstructed in the main tracker through the setup (right panel of Fig. 1).

In the following, we present results of physics performance studies in this detector system. They are based on the simulation of signals embedded into typical background events generated by UrQMD [2]. If not mentioned otherwise, all results refer to the collision system $\mathrm{Au}+\mathrm{Au}$ at $25 \mathrm{~A} \mathrm{GeV}$ beam momentum. The results were obtained with full detector response simulation and event reconstruction.

\section{Identification of kaons and particle ratio fluctuations}

Charged kaons, the most abundant strange particle species, will be separated from pions and protons by means of a time-of-flight measurement. The TOF wall at about $10 \mathrm{~m}$ distance from the target covers approximately $150 \mathrm{~m}^{2}$ and consists of RPC detectors with 

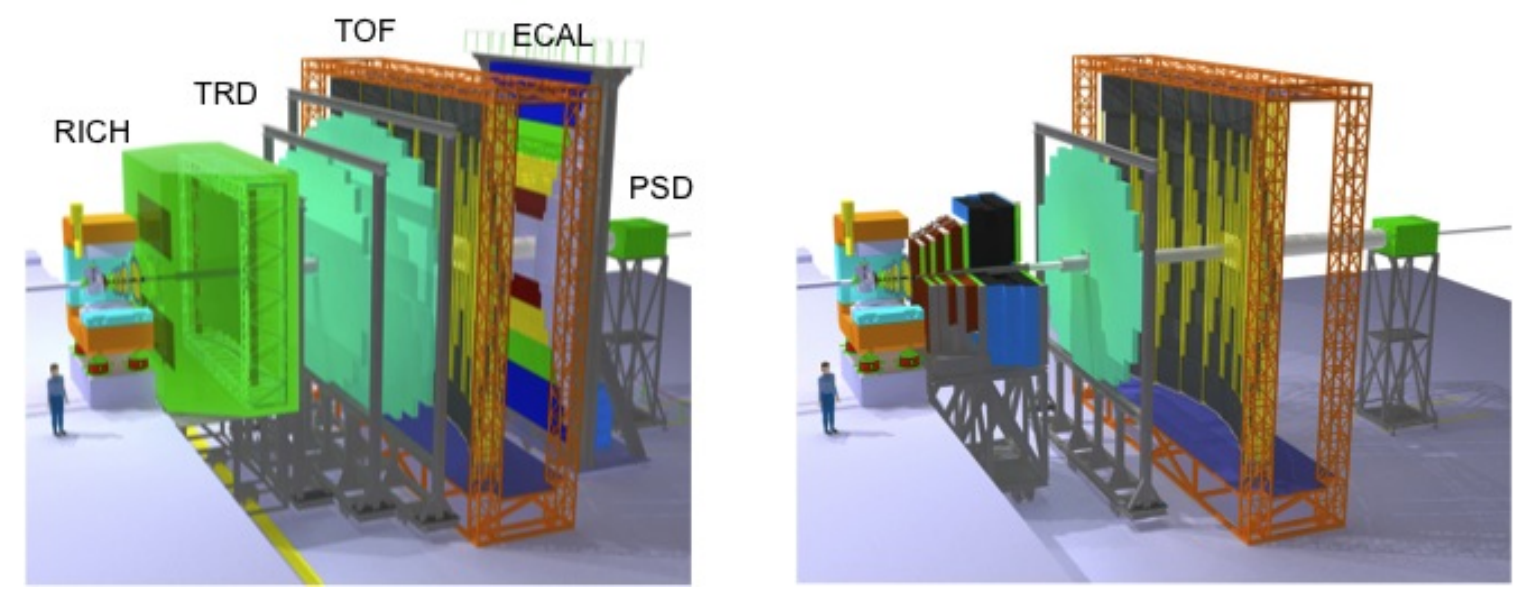

Figure 1. CBM experimental setup for (left) electron and hadron measurements and (right) muon measurements

pad and strip read-out. The goal of the detector development is to achieve a uniform system resolution of $80 \mathrm{ps}$, which will allow a clean kaon identification up to $3.5 \mathrm{GeV}$ as demonstrated in Fig. 2, covering the bulk of the produced kaons in the forward rapidity hemisphere and enabling to reliably extract full phase-space kaon yields.
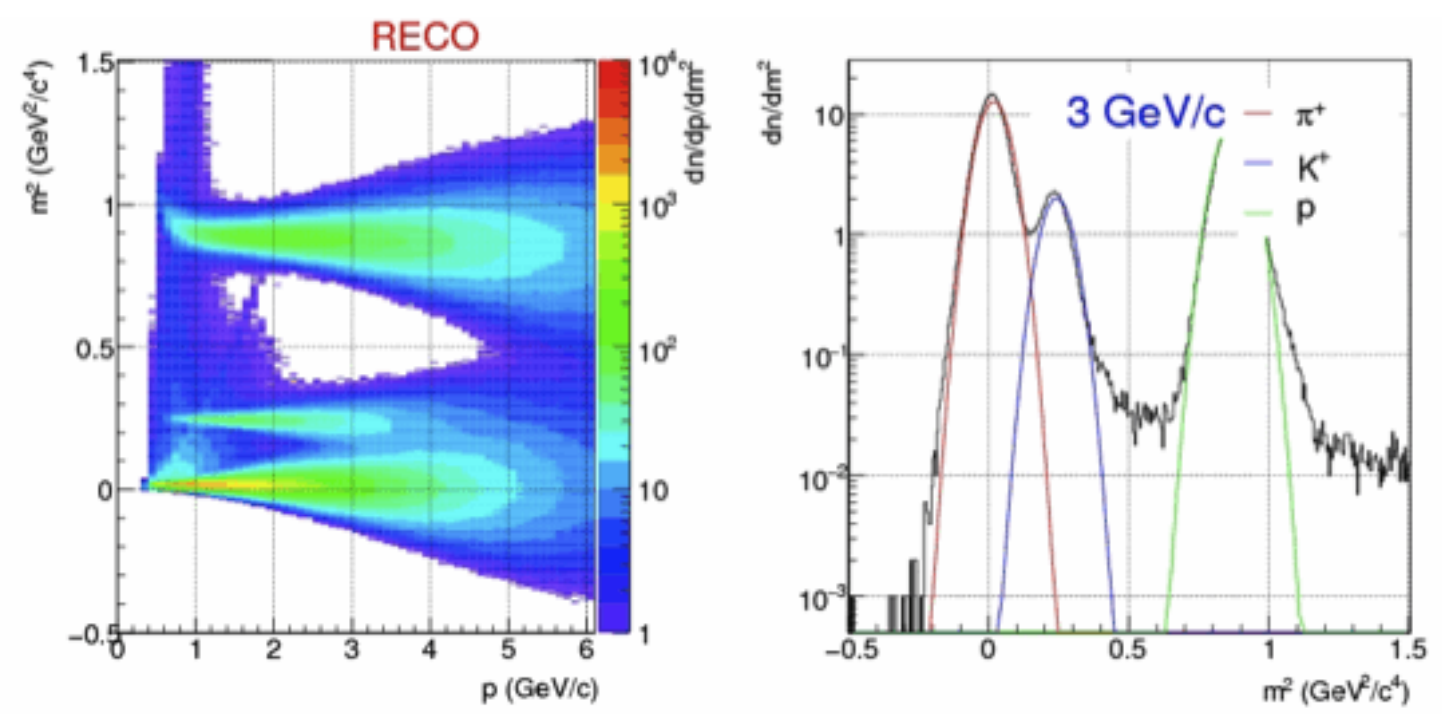

Figure 2. (Left) Reconstructed squared mass of hadrons as measured in the CBM time-of-flight system versus momentum. (Right) Squared-mass spectrum at $p=3 \mathrm{GeV}$.

Event-by-event fluctuations in the strange to non-strange particle yield ratios are discussed as a possible signature of the QCD critical end-point [4]. We thus investigated the sensitivity of CBM to such kind of fluctuations in terms of the measure $\sigma_{d y n}$ denoting the relative width of the distribution of the eventwise $K / \pi$ ratio after geometrical subtraction of the contributions from finite statistics and detector resolution, which are obtained by event mixing. As Fig. 3 shows, the experimental acceptance, track 
reconstruction and particle identification in CBM do not introduce a significant bias to the measurement; although the mean value and the total width of the input distribution generated by UrQMD change considerably, $\sigma_{d y n}$ is reproduced quite satisfactorily.
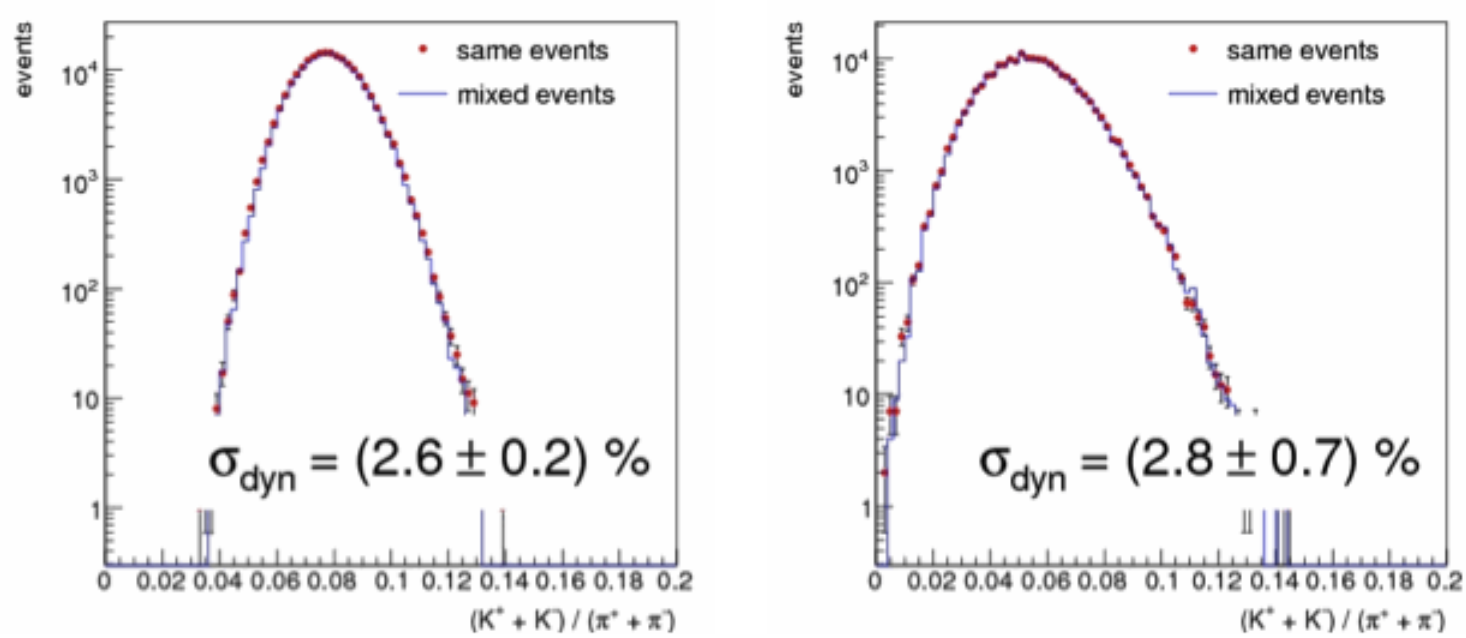

Figure 3. Simulated event-by event $K / \pi$ ratio in full phase space (left) and measured in the CBM acceptance (right)

\section{Hyperons and multi-strange di-baryons}

Hyperons will be measured by means of the reconstruction of their weak decay topology in the STS. The high accuracy of the track reconstruction in the silicon detectors results in an almost background-free measurement of the decays $\Lambda \rightarrow p \pi^{-}$and $\Xi^{-} \rightarrow \Lambda \pi^{-}$and a very good signal-to-background ratio for $\Omega^{-} \rightarrow \Lambda K^{-}[3]$. The same method also allows to address the hypothetical multi-strange di-baryons. As an example, we studied the decay $\left(\Xi^{0}, \Lambda\right) \rightarrow \Lambda \Lambda$ in the CBM-STS. We assumed a mass at the threshold, a lifetime of $3 \mathrm{~cm}$ and a branching ratio of $2 \%$ [5]. For the multiplicity, we employ the prediction of the thermal model $\left(7 \cdot 10^{-3}\right.$ in central $\mathrm{Au}+\mathrm{Au}$ collisions at $25 \mathrm{~A} \mathrm{GeV}$ beam momentum [6]). After appropriate topological cuts, in particular requiring the $\Lambda$ daughters not to point back to the interaction point, we arrive at a very clean signal in the invariant-mass spectrum $(S / B \approx 200$, see left panel of Fig. 4$)$. The sensitivity limit is only reached at a multiplicity three orders of magnitudes below the thermal model prediction (right panel of Fig. 4). CBM is thus well suited for the search for such exotic objects.

\section{Open charm}

The measurement of the hadronic decays of open charm in the CBM energy regime constitutes a particular challenge because of the very low multiplicity of charmed hadrons. For a sufficient suppression of the combinatorial background, the detection 

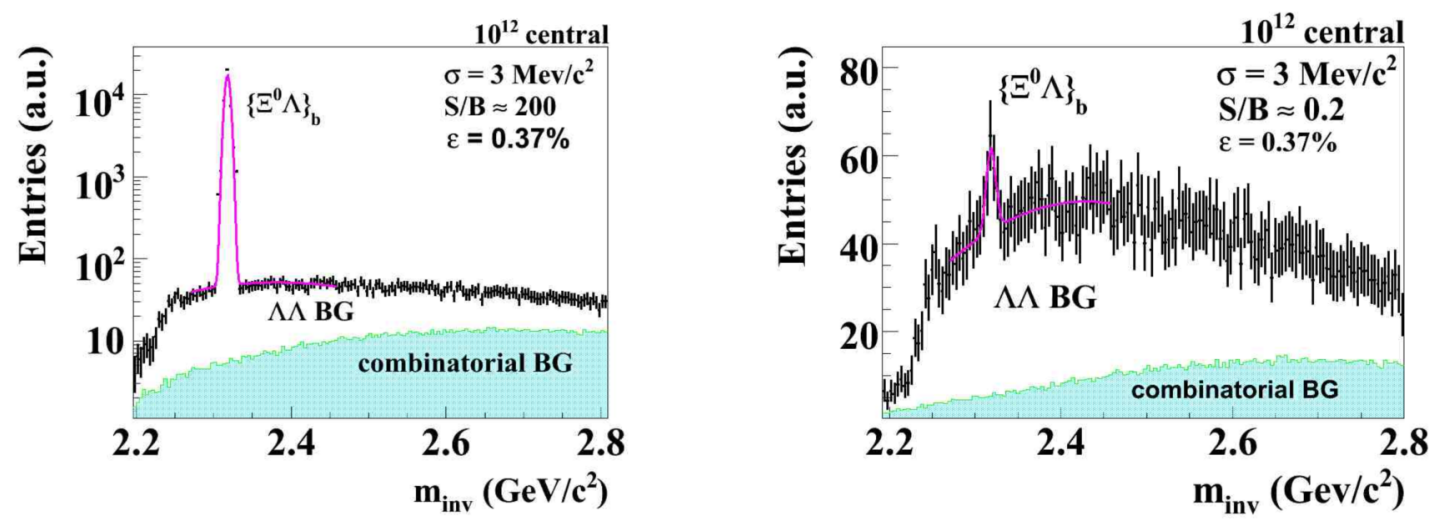

Figure 4. Invariant-mass signal of the decay $\left(\Xi^{0}, \Lambda\right) \rightarrow \Lambda \Lambda$, simulated for central $\mathrm{Au}+\mathrm{Au}$ collisions at $25 \mathrm{~A} \mathrm{GeV}$ with $\mathrm{CBM}$. The left hand panel shows the signal assuming a multiplicity as predicted by the statistical model. In the right-hand plot, the multiplicity is assumed to be three orders of magnitude smaller. The statistics of $10^{12}$ central events corresponds to 25 days of data taking at $10 \mathrm{MHz}$ interaction rate.

of the displaced decay vertices within a millimeter or less from the interaction point is indispensable. In CBM, a high-precision Micro-Vertex Detector (MVD) will be devoted to this task, using Monolithic Active Pixel Sensors with a single-hit resolution of about $3 \mu \mathrm{m}$ and low material budget. Two station of these detectors will be operated at $5 \mathrm{~cm}$ and $10 \mathrm{~cm}$ from the target, respectively. The read-out speed of the devices limits the maximal interaction rate for open charm measurements to $10^{6} / \mathrm{s}$. Simulations show that with this detector, a secondary-vertex resolution of about $50 \mu \mathrm{m}-80 \mu \mathrm{m}$, depending on the decay channel under study, can be achieved (see Table 1). This precision allows a clean separation of signal from background as shown in Fig. 5 for the decay channels $D^{0} \rightarrow K^{-} \pi^{-} \pi^{+} \pi^{+}$and $\Lambda_{c}^{+} \rightarrow p K^{-} \pi^{+}$. In terms of signal-to-background ratio, the fourparticle decay channel of the $D^{0}$ turns out to be favourable over the two-particle decay, since the geometrical and topological constraint of four tracks coming from the same origin is strict enough to overcome the background from four-particle combinatorics. Remarkably, even the measurement of $\Lambda_{c}$ seems to be in reach, which would give access to the total charm production.

Figure 6 shows the measured yield of $D^{+} \rightarrow K^{-} \pi^{+} \pi^{+}$as expected in a typical CBM run of 25 days at $1 \mathrm{MHz}$ interaction rate, as function of transverse momentum and rapidity. The coverage in both kinematical variables is sufficient to extract phasespace integrated yields. Table 1 summarises the properties of the so far studied open charm decay channels, assuming multiplicities as predicted by the HSD [7] model. The statistical model predictions differ from those of HSD, most pronounced so for $D_{s}^{+}$, the measurement of which would thus allow to discriminate different production scenarios. 

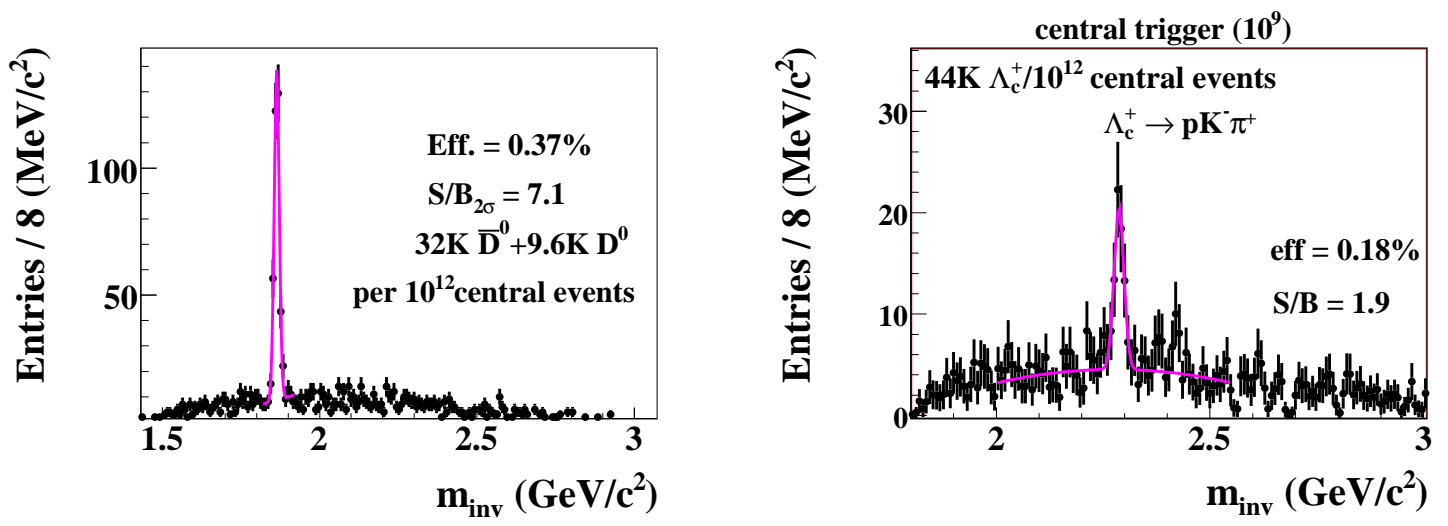

Figure 5. Invariant-mass signals of the decays $D^{0} \rightarrow K^{-} \pi^{-} \pi^{+} \pi^{+}$(left) and $\Lambda_{c}^{+} \rightarrow p K^{-} \pi^{+}$(right), obtained from simulation of central $\mathrm{Au}+\mathrm{Au}$ events at $25 \mathrm{AGeV}$ reconstructed with the $\mathrm{CBM}$ detector
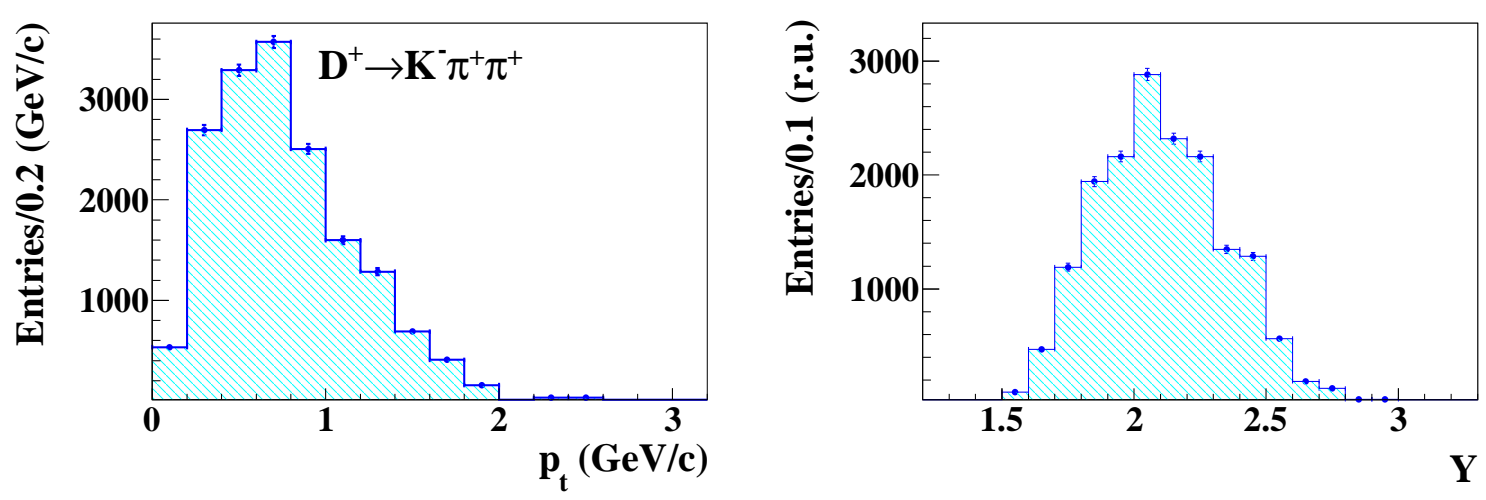

Figure 6. Expected yield of $D^{+}$detected in 25 days of CBM running Au+Au collisions at $25 \mathrm{~A} \mathrm{GeV}$, as function of transverse momentum (left) and rapidity (right)

\section{Charmonium}

CBM will measure charmonium in both the electron and the muon decay channel. For the electron case, the challenge is the separation of electrons from hadrons exploiting both the Cherenkov radiation in the RICH detector and the Transition Radiation in the TRD. The TOF and ECAL detectors can help for pion suppression at low and high momenta, respectively. Our simulations show that pion suppression factors of more than $10^{3}$ can be reached by combining the information from these detectors. Secondary electrons are suppressed by a cut on the transverse momentum. In the resulting invariant-mass spectrum (left panel of Fig. 7), the $J / \psi$ peak is well visible above the remaining background. A measurement of the $\psi^{\prime}$ might be feasible, but requires stronger cuts on the lepton candidates. A similar performance is obtained in the muon setup (right panel of Fig. 7). Here, the background mainly consists of wrong matches between tracks in the muon system and tracks reconstructed in the STS. 

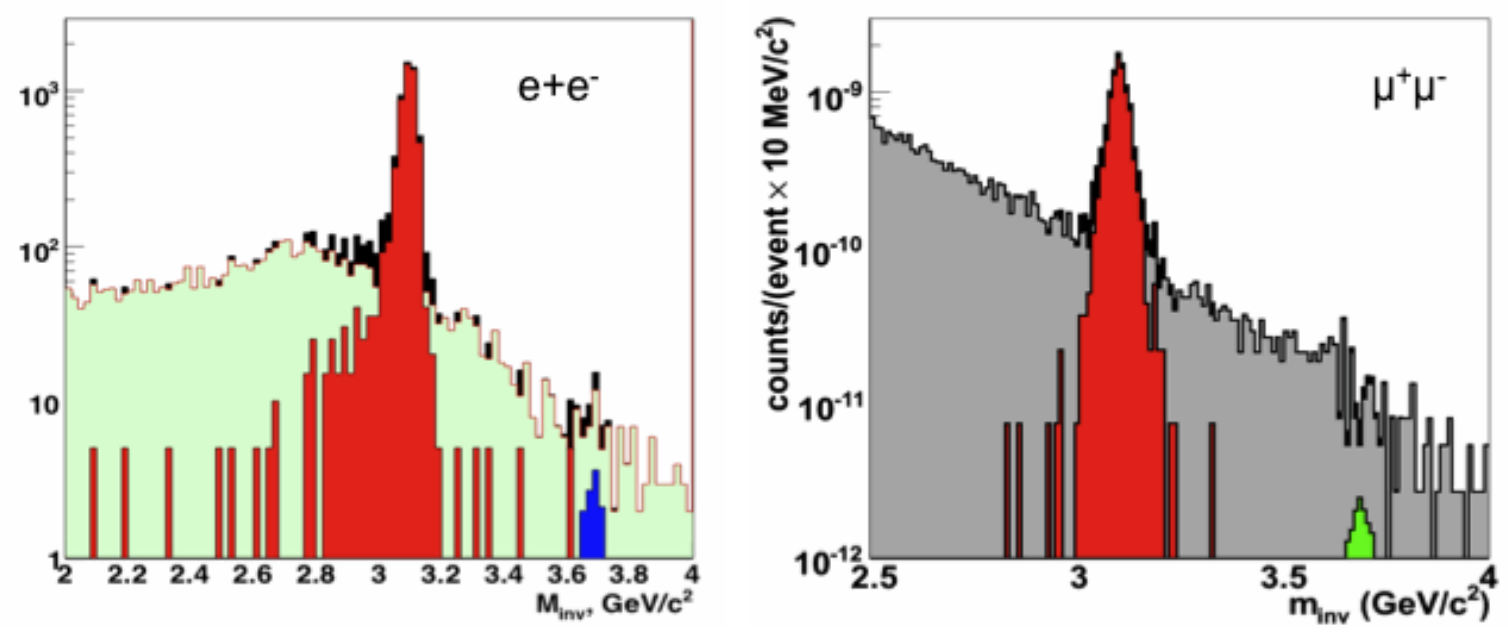

Figure 7. $J / \psi$ signal in the invariant-mass spectrum of electron pairs (left) and muon pairs (right), obtained from simulation of central $\mathrm{Au}+\mathrm{Au}$ events at $25 \mathrm{~A} \mathrm{GeV}$. The statistics corresponds to 25 days of data taking at $10 \mathrm{MHz}$ interaction rate.

\section{Challenges}

The high interaction rates needed for the charm measurements - up to $10 \mathrm{MHz}$ in the case of charmonium - pose extreme challenges not only to the radiation tolerance, rate capability and speed of the detectors, but also to the data handling. At $10^{7}$ interactions per second, a raw data rate of about $1 \mathrm{~TB} / \mathrm{s}$ has to be shipped from the front-ends and reduced online to a recordable rate of about $1 \mathrm{~GB} / \mathrm{s}$, i. e. by a about a factor of

Table 1. Properties of the reconstrution of open charm decays in the CBM experiment in central $\mathrm{Au}+\mathrm{Au}$ collisions at $25 \mathrm{~A} \mathrm{GeV}$. Shown are the predicted multiplicities by HSD [7] and by the Statistical Model, the branching ratios, the detection efficiency including geometrical acceptance, track reconstruction efficiency and analysis cuts, the secondary-vertex resolution, the resolution in invariant mass, the signal-to-background ratio, and the measured yield in $10^{11}$ central events, corresponding to about 25 days of CBM running at $1 \mathrm{MHz}$ interaction rate.

\begin{tabular}{cccccc}
\hline & $D^{0}+\overline{D^{0}}$ & $D^{0}$ & $D^{+}$ & $D_{S}^{+}$ & $\Lambda_{c}^{+}$ \\
decay channel & $K^{-} \pi^{+}+$c.c. & $K^{-} \pi^{-} \pi^{+} \pi^{+}$ & $K^{-} \pi^{+} \pi^{+}$ & $K^{-} K^{+} \pi^{+}$ & $p K^{-} \pi^{+}$ \\
$<n>_{\mathrm{HSD}}$ & $1.5 \cdot 10^{-4}$ & $4.0 \cdot 10^{-5}$ & $4.2 \cdot 10^{-5}$ & $5.4 \cdot 10^{-6}$ & \\
$<n>_{\mathrm{SM}}$ & $8.2 \cdot 10^{-4}$ & $2.0 \cdot 10^{-5}$ & $8.4 \cdot 10^{-5}$ & $1.4 \cdot 10^{-4}$ & $4.9 \cdot 10^{-4}$ \\
$\mathrm{BR}$ & $3.8 \%$ & $7.7 \%$ & $9.5 \%$ & $5.3 \%$ & $5.0 \%$ \\
Efficiency & $3.3 \%$ & $0.34 \%$ & $4.2 \%$ & $1.0 \%$ & $0.18 \%$ \\
vertex resolution & $54 \mu \mathrm{m}$ & $82 \mu \mathrm{m}$ & $60 \mu \mathrm{m}$ & $73 \mu \mathrm{m}$ & $70 \mu \mathrm{m}$ \\
$S / B$ & 4.4 & 7.1 & 9.0 & 0.3 & 1.9 \\
yield per runtime & $22 \mathrm{k}$ & $1 \mathrm{k}$ & $16 \mathrm{k}$ & $0.3 \mathrm{k}$ & $4 \mathrm{k}$ \\
\hline
\end{tabular}


1,000. This calls for a novel DAQ concept with self-triggered, free-streaming front-end electronics instead of a conventional trigger approach. A prototype of such a system is being developed at GSI and was tested under beam conditions with prototypes of the silicon strip detectors. Online event reconstruction will be performed in a dedicated computer farm consisting of a combination of FPGAs and modern many-core computers. The corresponding reconstruction algorithms are also being optimised with respect to speed, making use of the features of modern computer architectures (SIMD, many core, multi-threading). Currently, an event in the STS (about 600 charged tracks in the acceptance) can be reconstructed in about $50 \mathrm{~ms}$.

\section{References}

[1] Heuser J 2009 Nucl. Phys. A 830563 c

[2] Bass S et al. 1998, Prog. Part. Nucl. Phys. 41225

[3] Friese V 2006, J. Phys. G: Nucl. Phys. 32 S439

[4] Koch V 2008 J. Phys. G: Nucl. Phys. 35104030

[5] Schaffner-Bielich J, Mattiello R and Sorge H 2000, Phys. Rev. Lett. 844305

[6] Steinheimer J 2009, priv. comm.

[7] Linnyk O, Bratkovskaya E and Cassing W 2008, Int. J. Mod. Phys. E 171367 